\title{
A Turn for the Better - The Benefit of Doing A Limited Prone Rescan to Confirm the Exact Location of an Equivocal Vesicoureteric Junction Calculus Detected on Supine Abdominopelvic CT Scan
}

\author{
F. Rampersad, S. Persaud, A. Chan, J. Diljohn, P. Maharaj, and A. Ramsundar
}

\begin{abstract}
Background: In this case report, a calculus was seen at the vesicoureteric junction (VUJ) on computed tomography (CT) in an equivocal location. As subsequent urological management is based on the precise location of the calculus (ureteric orifice at the VUJ versus urinary bladder lumen) exact determination of its location is usually necessary. The simple manoeuvre of doing a limited prone $\mathrm{CT}$ rescan of the urinary bladder can precisely locate the calculus, thus allowing conservative or surgical management.

Case Presentation: A middle-aged male with a past history of urolithiasis presented with right sided abdominal pain, nausea and vomiting. Differentials included a right ureteric calculus and acute appendicitis. A CT scan of the abdomen and pelvis in the supine position revealed a $0.4 \mathrm{~cm}$ calculus at the expected position of the right $\mathrm{VUJ}$ with mild right hydronephrosis. As the exact location of this calculus could not be ascertained on supine imaging, a limited prone CT re-scan of the pelvis was subsequently performed, which confirmed the calculus within the urinary bladder, as it migrated to a dependent position in the lumen of the bladder on prone imaging. The patient was managed conservatively and passed the calculus via the urethra on the following day.

Conclusion: Prone CT is the gold standard for the evaluation of stone disease and can differentiate a vesical calculus from a vesicoureteric junction calculus. In cases where a supine abdominopelvic $\mathrm{CT}$ is performed (e.g. in institutions which do not routinely scan in the prone position for urolithiasis or in cases where a supine scan is done to exclude other pathologies), an additional limited prone $\mathrm{CT}$ is needed in equivocal cases for a suspected VUJ calculus. This additional limited prone CT can prevent unnecessary urologic intervention.
\end{abstract}

Index Terms- Vesicoureteric junction (VUJ) calculus, Ureteric calculus, Obstructive uropathy.

\section{INTRODUCTION}

A calculus located at the vesicoureteric junction (VUJ) on computed tomography $(\mathrm{CT})$ can either lie at the ureteric orifice at the VUJ or within the lumen of the urinary bladder (in close approximation to the ureteric office, but beyond it). Urological management of these cases are based on the precise location of the calculus, in addition to size and other clinical features. The simple manoeuvre of performing a limited prone $\mathrm{CT}$ rescan of the urinary bladder can confirm the precise location of the calculus and thus allow conservative management versus urological intervention (such as ureteroscopy or retrograde stenting).

Published on May 31, 2020

Authors are with the University of the West Indies, Trinidad and Tobago. (corresponding e-mail: adrianchanrad@gmail.com)

\section{Case report}

A 55-year-old male, with a past history of previous urolithiasis, presented with sudden onset right sided abdominal pain, associated with nausea and vomiting. Right lower quadrant tenderness was elicited on physical examination, but there was no guarding or rebound tenderness. His blood pressure, pulse and temperature were within normal limits. No blood investigations were performed. Differential diagnoses included renal colic and acute appendicitis. His Kidney/Ureter/Bladder (KUB) radiograph revealed no abnormality. A non-contrast Computed Tomography (CT) scan of the abdomen and pelvis was subsequently requested.

The supine abdominopelvic CT scan revealed a $0.4 \mathrm{~cm}$ calculus at the expected location of the right vesicoureteric junction (VUJ) (Figure 1), with mild right hydronephrosis and right perinephric fat stranding (Figure 2). The appendix was normal (Figure 3).

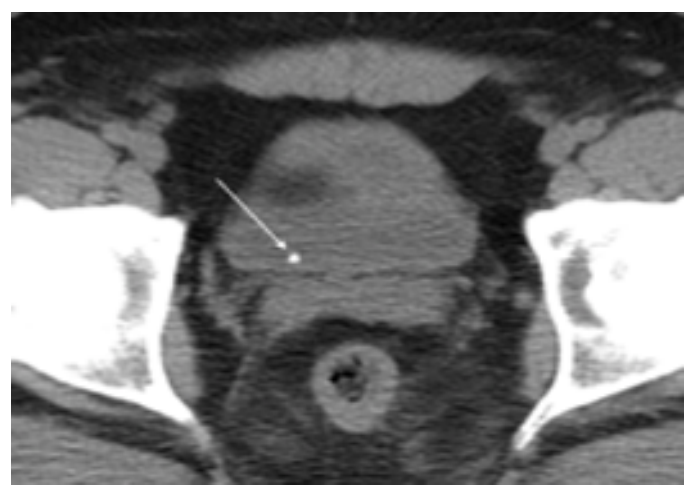

Fig 1: Supine axial non-contrast CT image showing a $0.4 \mathrm{~cm}$ calculus at the expected location of the right vesico-ureteric junction (arrow).

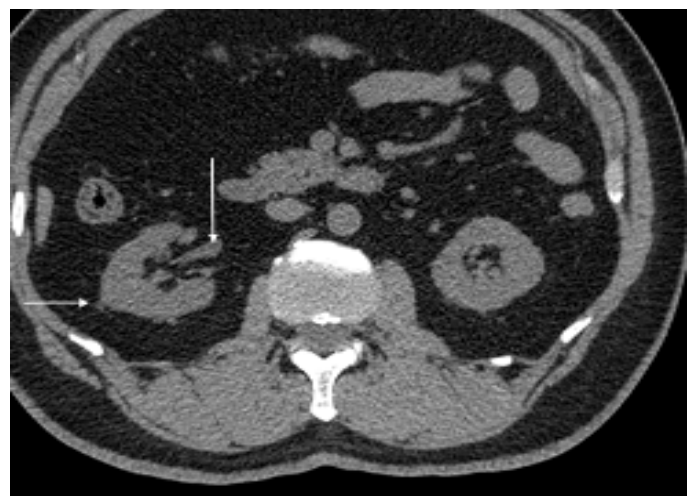

Fig 2: Mild right hydronephrosis, with a mildly dilated right renal pelvis (vertical arrow) and mild right perinephric stranding (horizontal arrow). 


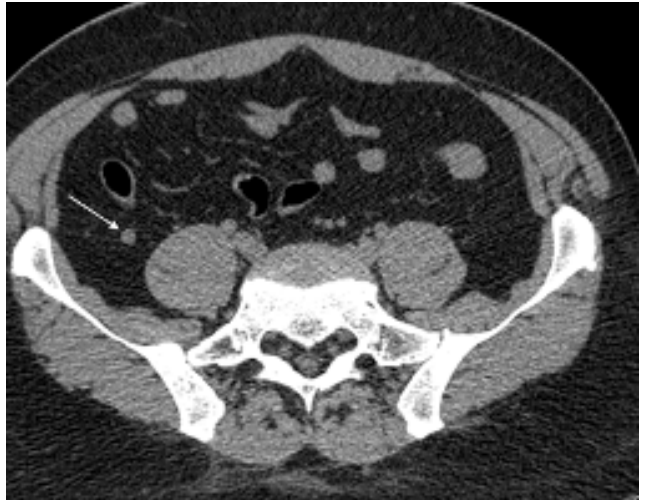

Fig 3: Normal retrocaecal appendix (arrow) on supine axial CT

The radiographer on duty observed the calculus at the right VUJ, and subsequently requested consent from the patient and supervising Radiologist to perform a limited rescan of the urinary bladder in the prone position. This prone scan revealed a vesical calculus rather than a VUJ calculus (Figure 4), confirmed by the movement of the calculus to a dependent position in the bladder lumen on the prone imaging.

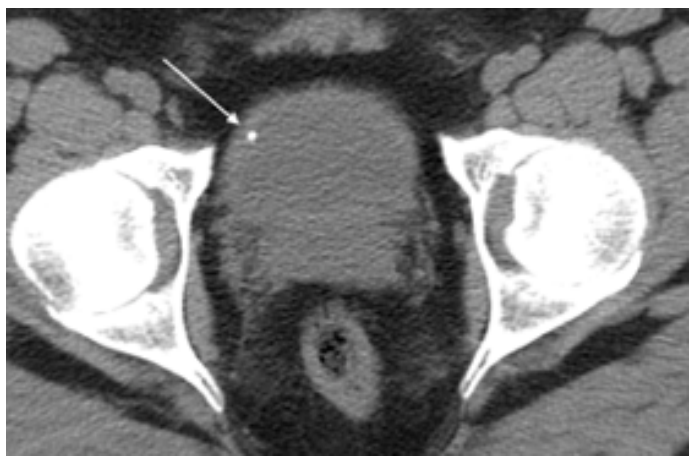

Fig 4: Axial non-contrast prone CT image (rotated to anatomical position) showing the calculus in a dependent position in the bladder lumen (arrow), confirming that this calculus is not lodged at the right vesicoureteric junction.

\section{DISCUSSION AND CONCLUSION}

CT scanning is regarded as the gold standard in the evaluation of patients with suspected urolithiasis.[1][2] It has a specificity of $100 \%$, sensitivity of $96 \%$ and a positive predictive value of $100 \%$, hence providing the physician with the confidence necessary to attribute a patients' symptoms to stone disease while excluding other pathologies.[3] A CT scan of the abdomen and pelvis, rather than a $\mathrm{CT}$ of the renal tract (CT kidneys, ureters and urinary bladder or CT KUB), was performed in our patient, as there was also the clinical suspicion of acute appendicitis.

Researchers have estimated that between $18-37 \%$ of patients presenting with flank pain have obstructing ureteric calculi located at the VUJ.[4] Challenges can sometimes arise in differentiating a VUJ calculus from a vesical calculus, if the latter is located in close approximation to the VUJ. Distinction between a VUJ calculus and bladder calculus on imaging is crucial, as this determines further management. Whenever a calculus is suspected at the VUJ in a supine CT scan, further imaging can be done in the prone position to help differentiate an intramural calculus at the ureteric orifice from a calculus that may have passed into the urinary bladder, as noted in our case.[4] The latter is more likely to be extruded spontaneously without intervention.

At some institutions, only prone CT scans of the abdomen and pelvis are performed for suspected urolithiasis, but this has not yet gained widespread acceptance.[5] More commonly, a scan is done in the supine position and an additional limited prone rescan is performed if a VUJ calculus is suspected.[6] This method can however result in misdiagnosis if the need to perform an additional prone scan is not recognized, which can then lead to unnecessary cystoscopy with a view to ureteric stenting, and its associated complications including urinary tract infections (UTIs), trauma or gross haematuria.[4]

Currently, the National Institute for Health and Care Excellence (NICE) and the European Association of Urology (EUA) do not have any guidelines with respect to supine versus prone CT imaging for suspected urolithiasis. It is however stated that protocols should be low dose and non-contrast. While the limited prone rescan entails an additional radiation dose, the dose employed in our case was small $(5.8 \mathrm{mSv})$ and below the European guidelines for a CT scan of the pelvis as stated by Rodrigues SI et al. [7] The effective dose recorded from our supine scan was $10.1 \mathrm{mSv}$.

It is hence necessary for the clinician, radiographer and supervising Radiologist to be aware of the need to perform a limited prone rescan through the urinary bladder in equivocal cases of a suspected vesicoureteric junction calculus. This is particularly important in supine abdominopelvic CT scans where the scan is performed for other suspected pathologies, and in institutions that do not routinely perform prone $\mathrm{CT}$ for suspected urolithiasis. The additional prone rescan can reduce misdiagnosis, unnecessary treatment and accelerate the diagnosis of a vesical calculus versus a VUJ calculus. Consideration should also be given to scanning suspected and known urolithiasis cases in the prone position, from the onset.

\section{REFERENCES}

[1] Ather MH, Memon W, Aziz W, Sulaiman MN. Non-contrast CT in the Evaluation of Urinary Tract Stone Obstruction and Haematuria. In Computed Tomography-Advanced Applications 2017 Aug 9. IntechOpen.

[2] Brisbane W, Bailey MR, Sorensen MD. An overview of kidney stone imaging techniques. Nature Reviews Urology. 2016 Nov;13(11):654.

[3] Kambadakone AR, Eisner BH, Catalano OA, Sahani DV. New and evolving concepts in the imaging and management of urolithiasis: urologists' perspective.

[4] Meissnitzer M, Meissnitzer T, Hruby S, Hecht S, Gutzeit A, HolzerFrühwald L, Hergan K, Forstner R. Comparison of prone vs. supine unenhanced CT imaging in patients with clinically suspected ureterolithiasis. Abdominal Radiology. 2017 Feb 1;42(2):569-76.

[5] Ather MH, Memon W, Aziz W, Sulaiman MN. Non-contrast CT in the Evaluation of Urinary Tract Stone Obstruction and Haematuria. InComputed Tomography-Advanced Applications 2017 Aug 9. IntechOpen.

[6] Levine J, Neitlich J, Smith RC. The value of prone scanning to distinguish ureterovesical junction stones from ureteral stones that have passed into the bladder: leave no stone unturned. AJR. American journal of roentgenology. 1999 Apr;172(4):977-81.

[7] Rodrigues SI, Abrantes AF, Ribeiro LP, Almeida RP. Dosimetry in abdominal imaging by 6-slice computed tomography. Radiologia Brasileira. 2012 Dec;45(6):326-33. 


\section{ABBREVIATIONS AND ACRONYMS}

VUJ: Vesicoureteric junction

\section{CT: Computed Tomography}

\section{AVAILABILITY OF DATA AND MATERIALS}

The case notes, images and consent form can be found at the Radiology Department, Gulf View Medical Center.

\section{ACKNOWLEDGMENT}

Gulf View Medical Centre and their Radiology and Urology Department, including Ms. Alida Ali and Dr. Michael Rampaul.
Author Information

\section{A. AFFILIATIONS}

1. Radiology Unit, Department of Clinical Medical Sciences, St Augustine Campus, University of the West Indies, Trinidad

○ Fidel Rampersad

O Paramanand Maharaj

- Adrian Chan

○ Jason Diljohn

- Ashton Ramsundar

2. Urology Unit, Department of Clinical Surgical Sciences, St. Augustine Campus, University of the West Indies, Trinidad. - Satyendra Persaud

3. Radiology Department, Gulf View Medical Center, Trinidad. ○ Sukhdev Singh

\section{B. CONTRIBUTIONS}

AC and JD contributed to acquisition of data and drafting the manuscript, SS obtained consent and. FR and SP did the revisions. All six authors read and approved the final manuscript. 